\title{
Thrombotic Occlusion of a Microvascular Anastomosis in a Resistance to Activated Protein C (APC) Patient with Incomplete Wound Healing after High Doses of Ascorbic Acid (Vitamin C)
}

\author{
Martijn P. J. Loonen", Bob De Frene, Bob Casaer
}

Department of Plastic, Reconstructive and Aesthetic Surgery at the Sint-Jan Hospital, Bruges, Belgium. Email: *mloonen@yahoo.com

Received December 21 $1^{\text {st }}$, 2011; revised January $10^{\text {th }}$, 2012; accepted February $7^{\text {th }}, 2012$

\begin{abstract}
A 45-year-old woman underwent a delayed breast reconstruction with a free Deep Inferior Epigastric Perforator Flap (DIEP flap) with total flap failure on the fourth postoperative day. Hematological investigation to exclude thrombofilia revealed a resistance to activated protein C (APC) with a factor V Leiden heterozygous mutation. The postoperative course was further complicated by delayed wound healing probably due to ascorbic acid (Vitamin C) related cytotoxic activity to fibroblasts. The surgeon must be aware of the use of preoperative nutritional supplement administration among patients. Future cost-effectiveness analyses should be made to warrant preoperative thrombophilia screening to prevent free flap failures.
\end{abstract}

Keywords: Thrombotic Occlusion; Vitamin C; Activated Protein C

\section{Case Report}

A 45-year-old woman underwent a delayed breast reconstruction with a free Deep Inferior Epigastric Perforator Flap (DIEP flap) in our department. The surgical history reported an appendectomy 10 years ago and a mastectomy 2 years ago for an invasive ductal carcinoma with a positive sentinel node biopsy followed by axillary lymph node dissection. No postoperative complications occurred during these procedures.

She reported a superficial vein thrombophlebitis in one leg during her chemotherapy.

Her medicine history reported no medication administration during the last 6 months preoperatively and no smoking history.

During the surgical procedure, we found an arterial spasm after the microsurgical anastomosis. Exploration of the arterial anastomosis revealed no thrombus and a new second anastomosis was performed without further surgical complications.

Postoperative intravenous Piracetam (Nootropil; 12 gram/24 hours) and subcutaneous Nadroparine injections (Fraxiparine; $2850 \mathrm{IE}$ anti-Xa (= $0.3 \mathrm{ml} / 24$ hours) were administered.

Postoperative flap monitoring during the first three

\footnotetext{
"Corresponding author.
}

postoperative days revealed no signs of flap ischemia or venous congestion.

On the fourth postoperative day however, routine clinical evaluation of flap vitals showed severe congestion of the whole flap (Figure 1). Emergency surgery with re-exploration revealed a venous thrombus with severe congestion injury of the flap tissues. Trombectomy was performed after which the flap was infused with a trombolytic agent (Urokinase). The vascular microanastomosis was revised and an arterial interposition graft was used to support the venous anastomosis.

A clinical re-occlusion of the vascular anastomosis was found 6 hours after the revision.

We decided to amputate the flap in consultation with the patient.

Partial central abdominal wound dehiscence started on day 5 post-operatively with abnormal amounts of wound fluid drainage based on fat necrosis (Figure 2). Secondary infection with non-fragilis bacteriodes was found and intravenous amoxicillin with clavulanic acid was started. Progressive necrosis and dehiscence happened until 14 days after the operation after which wound stabilization occurred.

Hematological investigation after the flap amputation, to exclude thrombofilia, revealed a resistance to activated protein C (APC) with a factor V Leiden heterozygous 


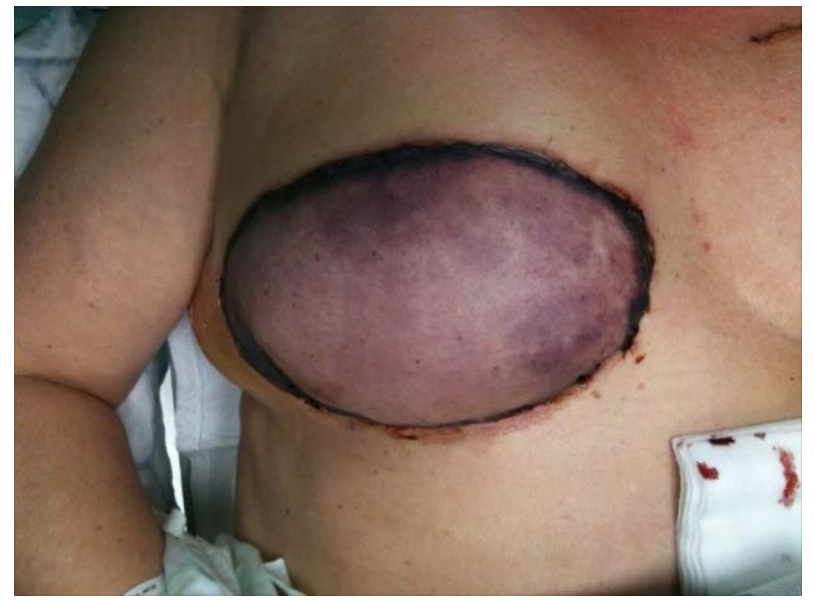

Figure 1. Severe congestion of the whole flap on the fourth postoperative day.

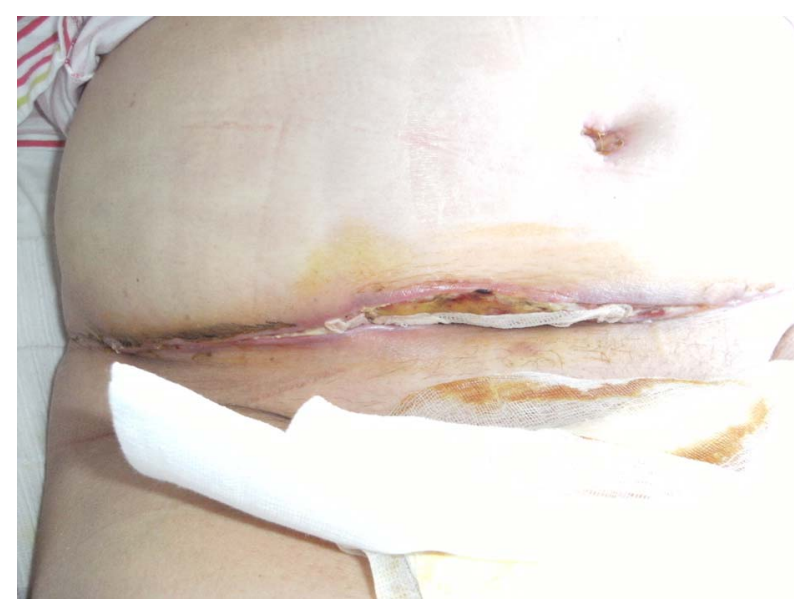

Figure 2. Partial central abdominal wound dehiscence with fat necrosis.

mutation. A slight increase of anti-cardiolipin IgM was found probably due to the infection without an increased anti-cardiolipin IgG level. No signs of protein C, S or antithrombin III deficiency, lupus anticoagulant or a prothrombine G20210A mutation were found. The INR value, quick prothrombin time test and the activated partial thromboplastin time (APTT) were within the normal range.

During a bed site visit, we found an unexpected medication box with nutritional supplements. She denied using any medication or supplements because she believed that these types of supplements do not belong to pharmaceutical drugs. One of these nutritional supplements was a combination of vitamin C (ascorbic acid), lysine, proline and flavonoids. The other consisted vitamin $\mathrm{C}$ and $\mathrm{D}$, the vitamin $\mathrm{B}$ complex, amino acids, minerals and trace elements. Consequently, she had administered herself daily a total of 1872 percent or nearly 19 times of the recommended daily allowance (RDA) vitamin $\mathrm{C}$ during a period of 4 months.

Comparable high concentrations of nearly 700 percent or 7 times of the vitamin B complex RDA was used daily during the same period.

These products were recommended by an alternative doctor to increase the potential tumor cytotoxic effects of high cellular antioxidant concentrations.

\section{Discussion}

\subsection{Resistance to Activated Protein}

Resistance to activated protein C (APC) is the most common genetic risk factor associated with venous thrombosis. Most cases are due to a point mutation in the factor $\mathrm{V}$ gene (factor $\mathrm{V}$ Leiden $F V L]$ ), which subsequently prevents the cleavage and disruption of activated factor $\mathrm{V}$ by APC and thus promotes ongoing clot development. In approximately $3 \%-8 \%$ of white adults, this mutation is heterozygous, conferring a 5-fold increased lifetime risk of venous thrombosis compared with the general population [1].

Irreversible thromboses after microvascular anastomosis due to hereditary thrombophilia have been described in the literature [2,3]. Furthermore, we found a former comparable free DIEP flap failure after an irreversible venous thrombosis in another patient with an APC resistance operated in our department. Future cost-effectiveness analyses should be made to warrant preoperative thrombophilia screening to prevent free flap failures.

\subsection{Ascorbic Acid (Vitamin C)}

The former appendectomy and mastectomy wounds in this patient healed without complications and therefore we link the abdominal wound dehiscence to her 4 months of highly dosed Vitamin C administration.

Pharmaceutical dosis of ascorbic acid have been reported to exert cytotoxic and anticancer activity in vitro and in vivo [4]. Increased ascorbic acid concentrations are found in the extracellular fluid in animal models after intravenous administration of pharmacologic concentrations of vitamin $C$ while substantial lower concentrations are found in blood [5]. One proposed working mechanism involves direct cytotoxity mediated by accumulation of ascorbic acid radicals and hydrogen peroxide in the extracellular environment of tumor cells but also to fibroblasts which may contribute to incomplete wound healing [6,7]. This could be an explanation for the delayed wound healing in our patient.

Secondly, high doses of ascorbic acid suppress NO (nitric oxide) generation. The endothelium of blood vessels uses nitric oxide to signal the surrounding smooth muscle to relax, thus resulting in vasodilatation and increasing blood flow. This angiostatic effect of vitamin C 
may explain the arterial spasm after the microsurgical anastomosis during the surgical procedure [4]. However, the hypothesized tissue accumulation of vitamin $\mathrm{C}$ in this patient needs to be further proven by laboratory testing in human models. Nevertheless, we suggest stopping preoperative administration of high doses of nutritional supplements.

\section{Conclusion}

The surgeon must be aware of the use of preoperative nutritional supplement administration among patients. Future cost-effectiveness analyses should be made to warrant preoperative thrombophilia screening to prevent free flap failures.

\section{REFERENCES}

[1] D. L. Chengelis, P. J. Bendick, J. L. Glover, O. W. Brown and T. J. Ranval, "Progression of Superficial Venous Thrombosis to Deep Vein Thrombosis," Journal of Vascular Surgery, Vol. 24, No. 5, 1996, pp. 745-749. doi:10.1016/S0741-5214(96)70007-1

[2] E. Olsson and P. Höijer, "Activated Protein C Resistance Due to Factor V Leiden, Elevated Coagulation Factor VIII and Postoperative Deep Vein Thrombosis in Late Breast Reconstruction with a Free TRAM Flap: A Report of Two Cases," British Journal of Plastic Surgery, Vol.
58, No. 5, 2005, pp. 720-723. doi:10.1016/j.bjps.2004.12.024

[3] I. C. Warnecke, F. Kretschmer, S. Brüner, O. Frerichs and H. Fansa, "Hereditary Thrombophilia in Free Microvascular Flaps-A Case Report," Handchirurgie Mikrochirurgie Plastische Chirurgie, Vol. 39, No. 3, 2007, pp. 220-224. doi:10.1055/s-2007-965139

[4] N. A. Mikirova, T. E. Ichim and N. Riordan, "Anti-Angiogenic Effect of High Doses of Ascorbic Acid,” Journal of Translational Medicine, Vol. 6, 2008, pp. 50-65. doi:10.1186/1479-5876-6-50

[5] Q. Chen, M. G. Espey, A. Y. Sun, J. H. Lee, M. C. Krishna, E. Shacter, P. L. Choyke, C. Pooput, K. L. Kirk, G. R. Buettner and M. Levine, "Ascorbate in Pharmacologic Concentrations Selectively Generates Ascorbate Radical and Hydrogen Peroxide in Extracellular Fluid in Vivo," Proceedings of the National Academy of Sciences, Vol. 104, No. 21, 2007, pp. 8749-8754. doi:10.1073/pnas.0702854104

[6] B. Peterkofsky and W. Prather, "Cytotoxicity of Ascorbate and Other Reducing Agents towards Cultured Fibroblasts as a Result of Hydrogen Peroxide Formation," Journal of Cellular Physiology, Vol. 90, No. 1, 1977, pp. 61-70. doi:10.1002/jcp.1040900109

[7] H. D. Jampel, “Ascorbic Acid Is Cytotoxic to Dividing Human Tenon's Capsule Fibroblasts. A Possible Contributing Factor in Glaucoma Filtration Surgery Success," Archives of Ophthalmology, Vol. 108, No. 9, 1990, pp. 1323-1325. doi:10.1001/archopht.1990.01070110139038 